\title{
Aphakic Pupillary Block by an Intact Anterior Vitreous Membrane after Total Lens Extraction by Phacoemulsification
}

\author{
Michihiro Kono Akiko Ishida Sho Ichioka Masato Matsuo \\ Hiroshi Shimizu Masaki Tanito \\ Department of Ophthalmology, Shimane University Faculty of Medicine, Izumo, Japan
}

\section{Keywords}

Acute primary angle closure - Aphakic angle closure $\cdot$ Anterior hyaloid membrane ·

Vitrectomy $\cdot$ Intrascleral intraocular lens fixation

\section{Abstract}

An 85-year-old Japanese woman with acute primary angle closure in her right eye underwent cataract extraction. Because of the weakness of the Zinn's zonules, all of the lens tissue including the lens capsule was removed by phacoemulsification. Because of the absence of vitreous prolapse into the anterior chamber, vitrectomy was not performed. Nine days postoperatively, acute angle closure due to pupillary block by an anterior vitreous membrane developed. To resolve the pupillary block, anterior vitrectomy was performed on the same day. Postoperatively, her symptoms resolved, the anterior chamber deepened, and the intraocular pressure normalized. Although rare, acute angle closure due to pupillary block by an anterior vitreous membrane can occur after total lens extraction with phacoemulsification. If no vitreous prolapse occurs with total lens extraction, an intentional hyaloidotomy using an anterior vitreous cutter or iridectomy should be considered to avoid secondary angle closure.

\section{Karger"}




\section{Case Reports in Ophthalmology}

\section{Introduction}

Small incisional cataract surgery is performed increasingly more often as the initial treatment of acute primary angle closure (APAC) because cataract extraction may be better than laser peripheral iridectomy regarding subsequent intraocular pressure (IOP) control [1]. However, completion of the planned phacoemulsification and intracapsular lens (IOL) implantation is not always easy in such eyes because of the presence of corneal edema, incomplete pupil dilation, a small working space, and Zinn's zonule weakness; the secondary implantation of an IOL is required when the aphakic status remains after the initial cataract surgery. We report a case of aphakic acute angle closure recurrence in an eye after a total lens extraction was performed to treat APAC.

\section{Case Report}

An 85-year-old Japanese woman presented to our hospital for repeated episodes of intermittent ocular pain and blurred vision in her right eye (OD), headache, and nausea; the last episode occurred the day before the initial visit. At the initial visit, the best-corrected visual acuity (BCVA) levels were (0.2) OD and (0.8) in her left eye (OS), and the IOP values were $12 \mathrm{~mm} \mathrm{Hg} \mathrm{OD}$ and $10 \mathrm{~mm} \mathrm{Hg}$ OS. By slit-lamp, gonioscopic, and anterior-segment optical coherence tomography (AS-OCT, Casia 2; Tomey, Nagoya, Japan) examinations, conjunctival injection, corneal edema, Descemet membrane folds, shallow anterior chamber (AC), dilated pupil, nuclear cataract (Emery-Little III), and a closed angle (Shaffer $0-1$ ) were seen OD (Fig. 1a, b). The lens vault was $1.298 \mathrm{~mm}$ OD by AS-OCT. The axial length was 23.12 $\mathrm{mm}$, and lens thickness was $5.32 \mathrm{~mm}$ OD by optical measurement (OA-2000; Tomey). She was diagnosed with APAC OD, and small incisional cataract surgery and IOL implantation were planned for the day of the initial visit because another episode of an acute IOP elevation could occur soon. During phacoemulsification, due to weakness of the Zinn's zonules, all of the lens tissue including the lens capsule was removed (online suppl. Video. 1; for all online suppl. material, see www.karger.com/doi/10.1159/000520176). Because of the absence of vitreous prolapse into the $\mathrm{AC}$, no vitrectomy was performed. At the visit 6 days postoperatively, the BCVA was 0.2 with +12.0-diopter (D) correction, the IOP was $24 \mathrm{~mm} \mathrm{Hg} \mathrm{OD}$, and the AC depth was normalized.

Nine days postoperatively, she presented to the emergency room of our hospital for sudden onset of ocular pain OD and headache. The BCVA was hand motions, and IOP was $60 \mathrm{~mm} \mathrm{Hg} \mathrm{OD,} \mathrm{with} \mathrm{conjunctival} \mathrm{injection,} \mathrm{corneal} \mathrm{edema,} \mathrm{and} \mathrm{loss} \mathrm{of} \mathrm{AC} \mathrm{despite} \mathrm{the}$ aphakic status (Fig. 2a, b). High-magnification AS-OCT showed transparent tissue covering the pupillary region (Fig. 2c, d). Fundus examination showed no uveal effusion or hemorrhagic choroidal detachment. She was diagnosed with acute angle closure due to pupillary block by an anterior vitreous membrane OD. To resolve the pupillary block, an anterior vitrectomy was performed that day. Intraoperatively, using triamcinolone (MaQaid; Wakamoto Pharmaceutical, Tokyo, Japan), the intact anterior vitreous membrane was removed (online suppl. Video 2). Postoperatively, the symptoms resolved rapidly; the BCVA was 0.3 with +10.0-D correction, the IOP was $9 \mathrm{~mm} \mathrm{Hg}$ OD, and the AC depth was normal (Fig. 3a, b). One month after the anterior vitrectomy, intrascleral IOL fixation was performed OD. At the final visit to our hospital 2 months after the anterior vitrectomy, the BCVA was 0.4 with -1.0-D correction, and the IOP was $9 \mathrm{~mm} \mathrm{Hg} \mathrm{OD;} \mathrm{episodes} \mathrm{of} \mathrm{shal-}$ lowing of the AC or elevated IOP did not occur, and no peripheral anterior synechia was observed by AS-OCT (Fig. 4a, b).

\section{Karger'}



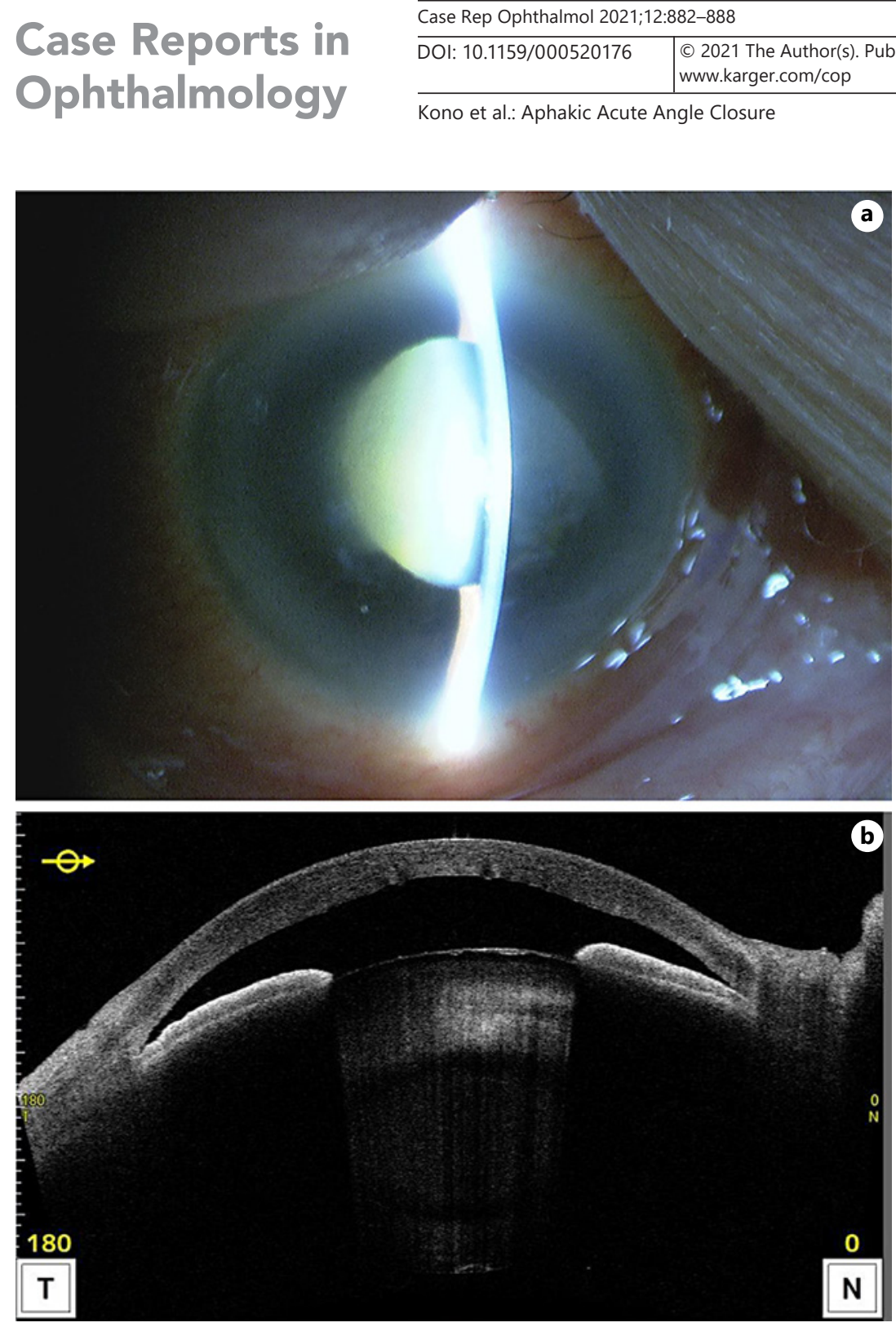

Fig. 1. Slit-lamp (a) and AS-OCT (b) findings OD at the initial visit. A shallow anterior chamber and closed angle are seen $(\mathbf{a}, \mathbf{b})$. The yellow arrow indicates the OCT scan direction. T, temporal; N, nasal.

\section{Discussion/Conclusion}

In the current case, the presence of thick lens (5.32 mm) [2] and lens vault (1.298 mm) [3] values suggested the lens involvement, that is, anterior displacement of lens and/or lens intumescent, in development of APAC and prompted us to perform an emergent cataract surgery. Because of the refined procedures and surgical skills associated with current phacoemulsification surgery, more surgeons are choosing primary cataract surgery as the initial treatment for APAC. Even in eyes with Zinn's zonule dialysis/weakness, total lens extraction can be completed by phacoemulsification with closed eye surgery as in the current case. In such cases, later implantation of an IOL with intrascleral fixation is the choice for visual recovery. After lens extraction, regardless of pseudophakia or aphakia, recurrent acute angle closure is unusual. Although acute aphakic pupillary block by an anterior vitreous membrane was reported in the eras of intracapsular cataract extraction [4] and extracapsular cataract extraction [5], a report of this etiology in the current era is unique in the literature. 

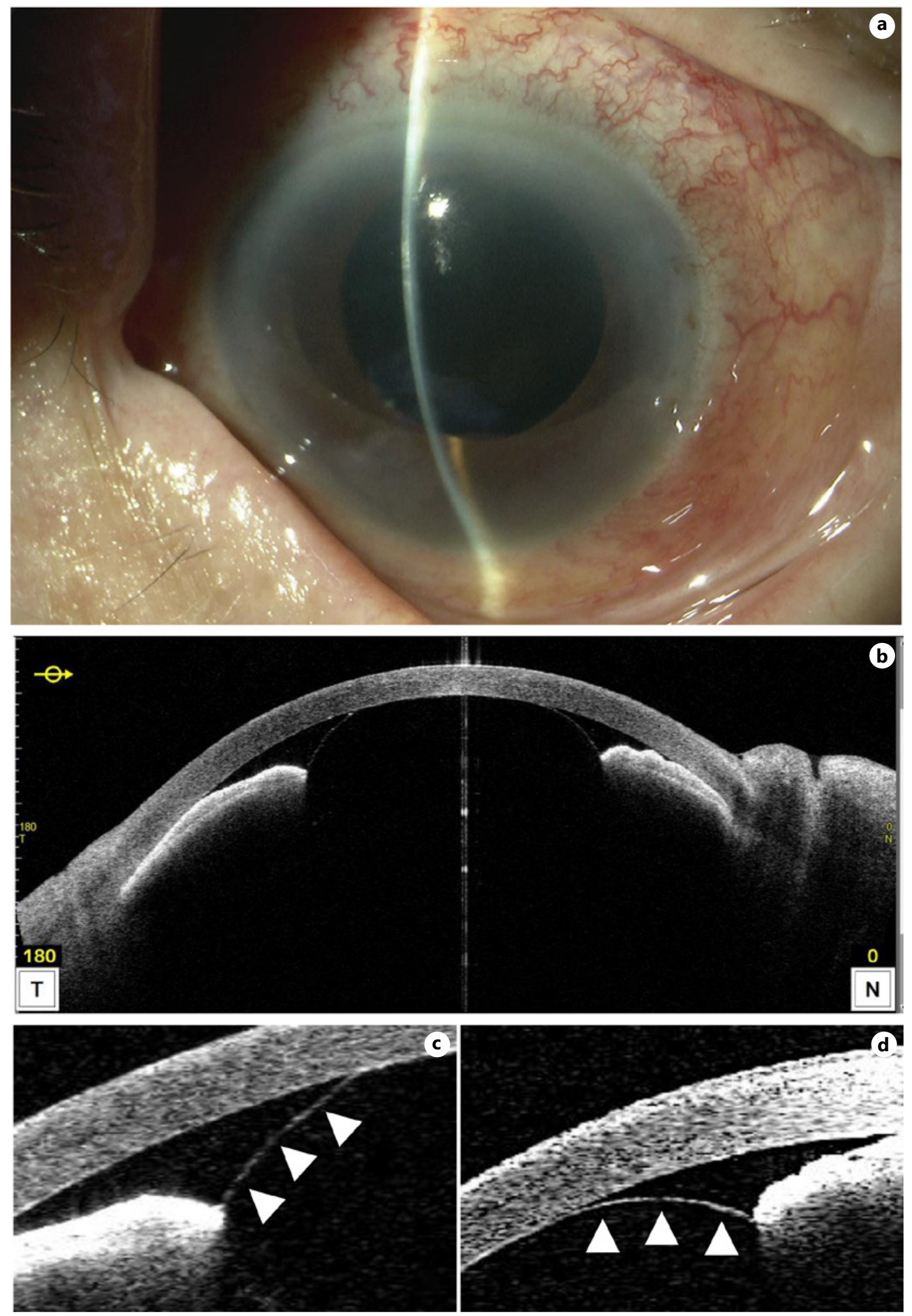

Fig. 2. Slit-lamp (a) and AS-OCT (b-d) findings OD 10 days after the total cataract extraction. A shallow anterior chamber and closed angle are seen despite the absence of the lens and lens capsule (a, b). AS-OCT shows an anterior vitreous membrane (arrowheads) in both the temporal (c) and nasal (d) scan directions. The yellow arrow indicates the OCT scan direction. $\mathrm{T}$, temporal; $\mathrm{N}$, nasal. 


\section{Case Reports in Ophthalmology}
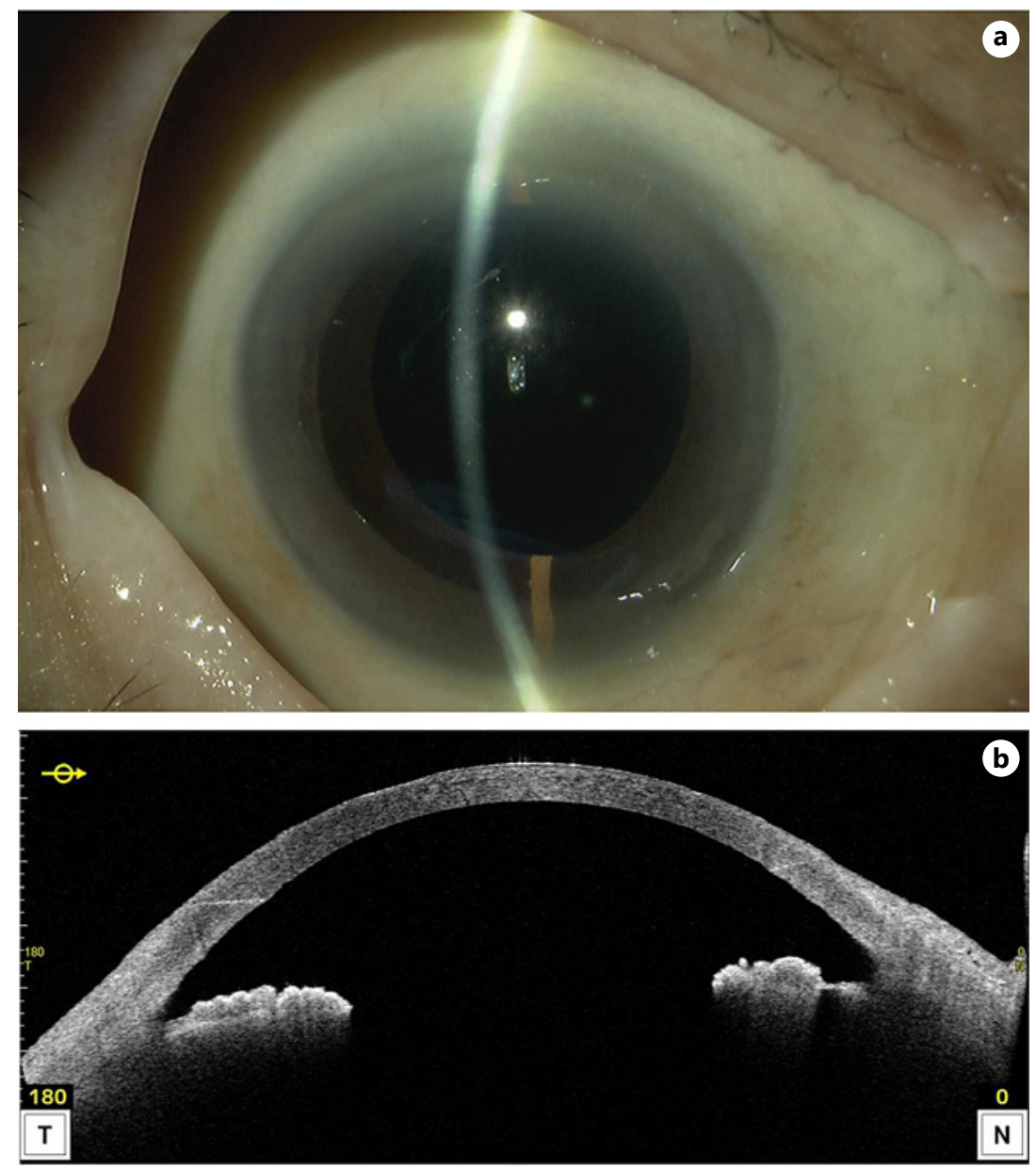

Fig. 3. Slit-lamp (a) and AS-OCT (b) findings OD 1 month after the vitrectomy. The deepened anterior chamber and open angle after anterior vitreous membrane are seen $(\mathbf{a}, \mathbf{b})$. The yellow arrow indicates the OCT scan direction. T, temporal; N, nasal.

During the initial phacoemulsification, no vitreous prolapse occurred despite the total lens extraction, probably due to the presence of dehiscence between the posterior lens capsule and anterior vitreous membrane, that is, dehiscence or weakness of Wieger's ligament, in our elderly patient [6]; as a result, the intact anterior membrane remained. Given the presence of the anterior vitreous membrane and vitreous gel, aqueous misdirection could have triggered and/or aggravated the angle closure in this case. Use of miotics may help reduce the anterior vitreous prolapse but can exacerbate the shallow AC and pupillary block and hinder a subsequent surgical approach if it failed. Use of mydriatics relieves the aqueous misdirection, although involvement of this mechanism was uncertain; mydriatics can exacerbate anterior vitreous prolapse. Accordingly, any medical management seemed inadequate for reducing the IOP and deepening the AC in our case. Although hyaloidotomy using a neodymium:yttrium-aluminum-garnet (Nd:YAG) laser is another treatment option for pupillary block due to an anterior vitreous membrane [7, 8], because the anterior vitreous membrane was extensively connected to the corneal endothelium (Fig. 2b), an anterior vitrectomy seemed safer than Nd:YAG laser treatment in this case. Although we did not do so in our case, before the anterior vitrectomy, an attempt to lower IOP by using systemic acetazolamide and/or mannitol might confer a safe surgery. 

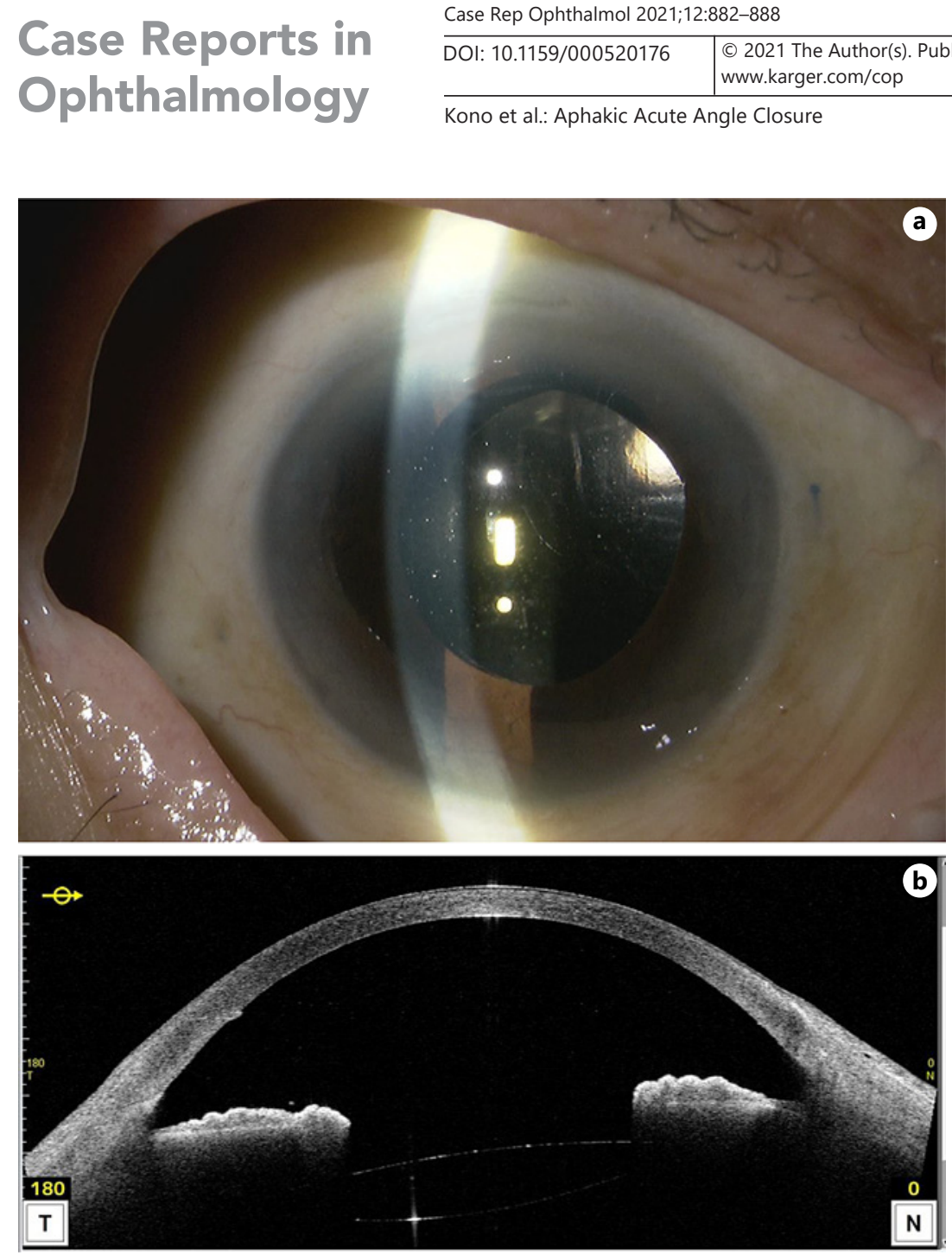

Fig. 4. Slit-lamp (a) and AS-OCT (b) findings OD 2 months after the intrascleral IOL fixation. The shallow anterior chamber or angle closure did not recur $(\mathbf{a}, \mathbf{b})$. The yellow arrow indicates the OCT scan direction. T, temporal; N, nasal.

In surgery for congenital/childhood cataract, posterior capsulotomy and anterior hyaloidectomy are the common procedures in combination with IOL optic capture when the IOL is implanted or iridectomy when the IOL is not implanted [9]. In adult cases, to avoid aphakic pupillary block, peripheral iridectomy during an ICCE procedure was performed routinely. However, modern surgeons may not pay close attention to this etiology after small incisional cataract surgery. In this context, our case is educational for modern surgeons.

In conclusion, although rare, acute angle closure due to pupillary block by an intact anterior vitreous membrane can occur after total lens extraction with phacoemulsification. Even if no vitreous prolapse occurs with total lens extraction, intentional hyaloidotomy using an anterior vitreous cutter or iridectomy should be considered to avoid secondary angle closure.

\section{Statement of Ethics}

This study adhered to the tenets of the Declaration of Helsinki. The Institutional Review Board of Shimane University Hospital did not require an ethics committee review process to report this case. The patient provided written informed consent for publication of this case report and any accompanying pictures. 


\section{Conflict of Interest Statement}

The authors have no conflicts of interest associated with this report.

\section{Funding Sources}

No financial support was provided.

\section{Author Contributions}

M.K., A.I., S.I., M.M., H.S., and M.T. treated the subject and collected the clinical data. M.K. and M.T. wrote the manuscript, and A.I., S.I., M.M., and H.S. revised the manuscript. All authors approved the final version of the manuscript. The authors agree to be responsible for all aspects of this work.

\section{Data Availability Statement}

All data generated or analyzed during this study are included in this article. Further enquiries can be directed to the corresponding author.

\section{References}

1 Husain R, Gazzard G, Aung T, Chen Y, Padmanabhan V, Oen FT, et al. Initial management of acute primary angle closure: a randomized trial comparing phacoemulsification with laser peripheral iridotomy. Ophthalmology. 2012;119(11):2274-81.

2 Wang Z, Chung C, Lin J, Xu J, Huang J. Quantitative measurements of the ciliary body in eyes with acute primaryangle closure. Invest Ophthalmol Vis Sci. 2016;57(7):3299-305.

3 Moghimi S, Fathollahzadeh N, Chen R, Lin SC, Weinreb RN. Comparison of Fellow eyes of acute primary angle closure and phacomorphic angle closure. J Glaucoma. 2019;28(3):194-200.

4 Hitchings RA. Acute aphakic pupil block glaucoma: an alternative surgical approach. Br J Ophthalmol. 1979; 63(1):31-7.

5 Van Buskirk EM. Pupillary block after intraocular lens implantation. Am J Ophthalmol. 1983;95(1):55-9.

6 Kawasaki S, Tasaka Y, Suzuki T, Zheng X, Shiraishi A, Uno T, et al. Influence of elevated intraocular pressure on the posterior chamber-anterior hyaloid membrane barrier during cataract operations. Arch Ophthalmol. 2011;129(6):751-7.

7 Epstein DL, Steinert RF, Puliafito CA. Neodymium-YAG laser therapy to the anterior hyaloid in aphakic malignant (ciliovitreal block) glaucoma. Am J Ophthalmol. 1984;98(2):137-43.

8 Fournié P, Malecaze F, Ponchel C, Arné JL. Late-onset hyaloideocapsular block syndrome. Ophthalmology. 2007;114(10):1839-41.

9 Self JE, Taylor R, Solebo AL, Biswas S, Parulekar M, Dev Borman A, et al. Cataract management in children: a review of the literature and current practice across five large UK centres. Eye. 2020;34(12):2197-218.

\section{Karger' ${ }^{\prime \prime}$}

